\title{
Polar vs. Nonpolar InGaN/GaN Quantum Heterostructures: Opposite Quantum Confined Electroabsorption and Carrier Dynamics Behavior
}

\author{
Emre Sari ${ }^{1}$, Sedat Nizamoglu ${ }^{1}$, Jung Hun Choi $^{2}$, Seung Jae Lee ${ }^{3}$, Kwang Hyeon Baik ${ }^{4}$, \\ In Hwan Lee ${ }^{2}$, Jong Hyeob Baek ${ }^{3}$, Sung-Min Hwang ${ }^{4}$ and Hilmi Volkan Demir ${ }^{1,5^{*}}$ \\ ${ }^{I}$ Department of Electrical and Electronics Engineering, Department of Physics, Nanotechnology Research Center, and National \\ Institute of Materials Science and Nanotechnology UNAM, Bilkent University, 06800 Bilkent, Ankara, Turkey; \\ ${ }^{2}$ School of Advanced Materials Engineering, Chonbuk National University, Chonju 561-756, Korea; \\ ${ }^{3}$ Korea Photonics Technology Institute, Gwangju,500-460 Korea; \\ ${ }^{4}$ Optoelectronics Lab., Korea Electronics Technology Institute, Seongnam, Gyeonggi 463-816, Korea; \\ ${ }^{5}$ School of Electrical and Electronic Engineering, Microelectronics Division; School of Physical and Mathematical Sciences, \\ Physics and Applied Physics Division, Nanyang Technological University, Singapore 639798
}

\begin{abstract}
We present a study of quantum confined electroabsorption and carrier dynamics in polar $c$-plane and nonpolar a-plane InGaN/GaN quantum heterostructures. We demonstrate red-shifting absorption edge, due to quantum confined Stark effect, in nonpolar InGaN/GaN quantum structures in response to increased electric field, while we show the opposite effect with blue-shifting absorption spectra in polar quantum structures. Moreover, confirmed by timeresolved photoluminescence measurements, we prove that carrier lifetimes increase with increasing electric field for nonpolar structures, whereas the opposite occurs for polar ones.
\end{abstract}

Since the demonstration of blue light emitting diodes [1] and laser diodes [2], optoelectronic devices based on IIINitride quantum structures have found important applications in consumer electronics and lighting industries through display, data storage and white-LED applications [3]. Moreover, in this semiconductor device platform, there is a great potential for even further expansion in optoelectronic device applications through innovation and development [4-5]. To widen their use, to improve their performances, and to expand their applications, physics of these devices should be understood well and materials quality and device efficiencies should be increased. In our research group, we have been working on the physics and applications of optoelectronic devices based on InGaN/GaN quantum structures. Here in this paper, we review the basic physics of InGaN/GaN quantum structures and present our studies on their external electric field dependent optical absorption and time resolved decay kinetics behavior.

\section{Polarization-induced built-in electrostatic fields}

In III-Nitride quantum heterostructures, two-dimensional built-in charges of alternating signs are induced at the interfaces due to the discontinuities of the polarization fields throughout the structure [6]. These charges cause built-in electrostatic fields, which are perpendicular to the interfaces and have alternating directions in quantum well and barrier layers in a multiple quantum well structure. This effect is maximal when the growth is performed on polar $c$-plane of III-N wurtzite crystal. As a consequence of this effect, electrons and holes are pulled in the opposite directions inside the well layers. This results with a reduced electronhole overlap and thus a reduced electron-hole recombination and generation rate compared to the no polarization induced electrostatic field case [4].

However, these fields and this effect are absent when the structure is grown on its $m$ - or $a$-plane [6]. These two planes are referred to as nonpolar planes. One of the drawbacks for growing III-Nitrides on nonpolar planes is that, dislocation densities in nonpolar-grown structures are much higher than those on polar structures, mainly due to the absence of a native substrate. And this makes them unfavorable for device applications. In a recent study, metal organic chemical vapor deposition (MOCVD) growth of $a$-plane $\mathrm{GaN}$ on $r$-plane sapphire substrate was achieved with a low dislocation density [7]. The authors also demonstrated a light emitting diode using this material.

Electroabsorption in III-Nitride Quantum Structures

As mentioned earlier, polarization induced electrostatic fields are present in polar III-Nitride quantum structures. The direction of these fields inside the quantum well layers is in opposite direction to the growth direction when the growth is performed using MOCVD. By placing these quantum structures in a $p-i-n$ diode architecture, in which $\mathrm{p}$ type layer is on top, external electric field can be applied in opposite direction to the built in electrostatic field inside the well layers. Then, an increasing external electric field decreases the net field inside the well layers, increasing the overlap integral of electron and hole wavefunctions. In addition to this, the tilt in the quantum well layers' potential

\footnotetext{
* e-mail: volkan@bilkent.edu.tr, hvdemir@ntu.edu.sg
} 
profile decreases and the energy difference between electron and hole ground states decreases. This effect can be probed through electroabsorption measurements and it is observed as a blueshift [5] in the absorption spectra at increasing external electric fields, i.e., reverse bias voltages, as in Figure 1.

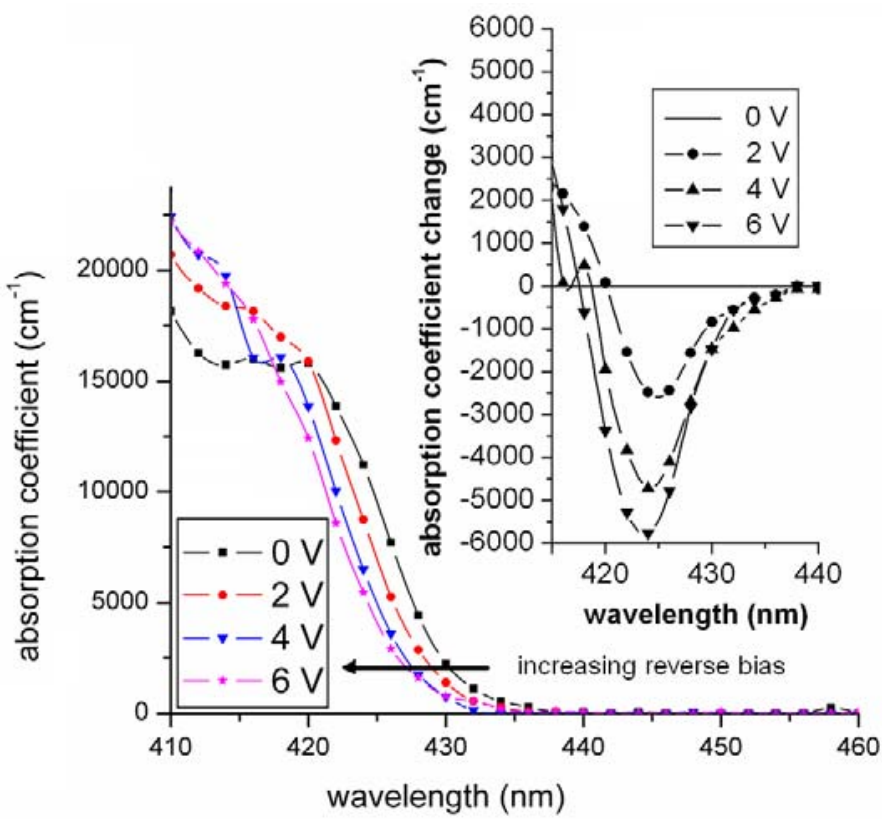

Fig. 1. Absorption spectra of polar InGaN/GaN quantum structures under different reverse bias voltages, the inset shows the absorption coefficient change relative to no-bias case.

The absorption coefficient change $(\Delta \alpha)$ in our InGaN/GaN quantum structures is significantly high, at a comparable level with other III-V quantum structures that operate at near infrared region and are widely used in telecom. This makes our devices promising candidates for their future use [5].

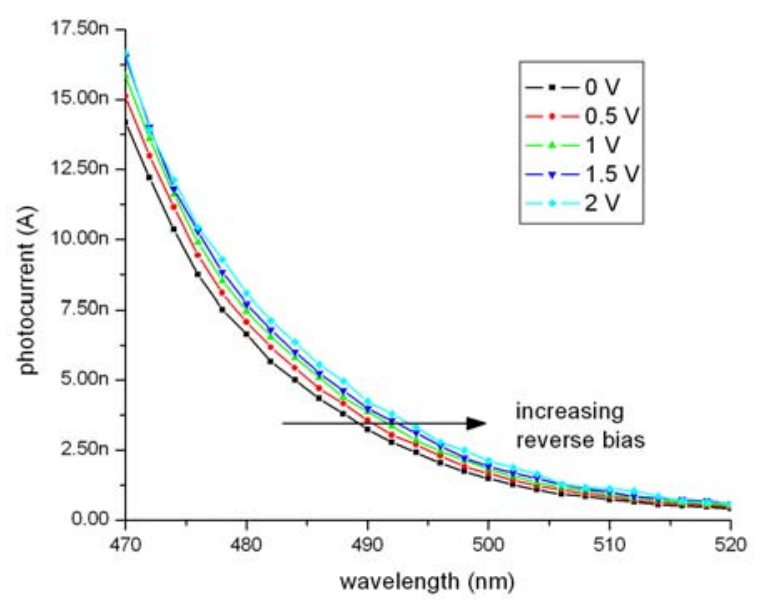

Fig. 1. Absorption spectra of nonpolar InGaN/GaN quantum structures under different reverse bias voltages. Absorption edges shift to longer wavelengths as the reverse bias is increased.
We also performed electroabsorption measurements in nonpolar structures. In this case, since we have no polarization-induced electrostatic fields inside the well layers, absorption spectrum of the device redshifts with increasing external electric field, a phenomenon known as quantum confined Stark effect [8].

The opposite behavior of electroabsorption in polar and nonpolar quantum structures stems from the existence of polarization induced electrostatic fields.

In addition to electroabsorption measurements, another characterization method is time-resolved photoluminescence. Due to the decrease of the overlap integral of electron and hole wavefunctions with increasing external electric fields, similar to the results of Ref. 9, we can observe a drastic decrease of carrier lifetimes in polar structures as in Fig. 3 [10].

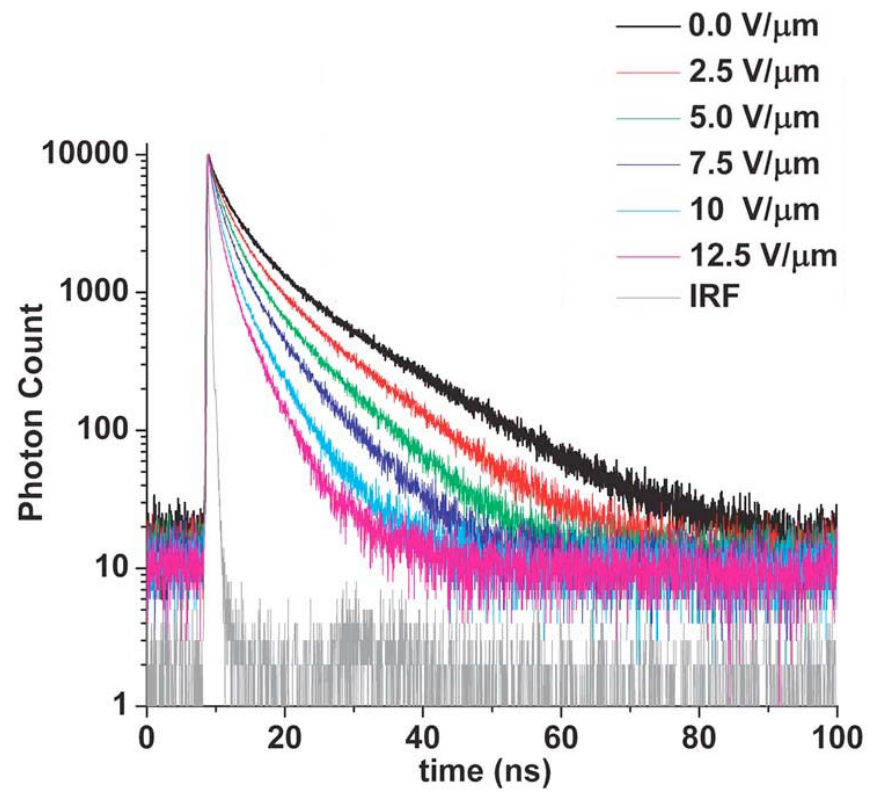

Fig. 3. Time resolved photoluminescence decays of polar InGaN/GaN quantum structures under different external electric fields, decays become faster as the external electric field increases.

An opposite behavior, increase of carrier lifetimes with increasing external electric field was observed (not shown) in the nonpolar case.

In conclusion, we reviewed the opposite behavior of electroabsorption and electric field dependent carrier lifetime behavior in polar and nonpolar InGaN/GaN quantum structures.

\section{REFERENCES}

[1] S. Nakamura, M. Senoh, N. Iwasa and S. Nagahama, Appl. Phys. Lett., 67, 1868 (1995).

[2] S. Nakamura, M. Senoh, S. Nagahama, N. Iwasa, T. Yamada, T. Matsushita, Y. Sugimoto and H. Kiyoku, Appl. Phys. Lett., 69, 4056 (1996).

[3] S. Nizamoglu, G. Zengin, and H. V. Demir, Appl. Phys. Lett., 92, 031102 (2008).

[4] J. Goldberger, R. He, Y. Zhang, S. Lee, H. Yan, H.-J. Choi and P. Yang, Nature, 422, 599 (2003). 
[5] E. Sari, T. Ozel, S. Nizamoglu and H. V. Demir, Appl. Phys. Lett., 90, 011101 (2007).

[6] C. Wetzel, M. Zhu, J. Senawiratne, T. Detchprohm, P.D. Persans, L. Liu, E.A. Preble, D. Hanser, Journal of Crystal Growth, 310, 17, 3987-3991 (2008).

[7] S.-M. Hwang,Y.-G. Seo, K.-H. Baik,I.-S. Cho, J.-H. Baek, S.-K Jung, T. G. Kim and M.-W. Cho, Appl. Phys. Lett., 95, 071101 (2009).

[8] D. A. B. Miller, D. S. Chemla, T. C. Damen, A. C. Gossard, W. Wiegmann, T. H. Wood, and C. A. Burrus, Phys. Rev. B 32, 1043 (1985).

[9] Y.-D. Jho, J.-S. Yahng, E. Oh, and D.-S. Kim, Phys. Rev. B, 66 0353334 (2002).

[10] E. Sari, S. Nizamoglu, I-H. Lee, J-H. Baek and H. V. Demir, Appl. Phys. Lett., 94, 211107 (2009). 